\title{
The high frequency variations in the rotation of Eros
}

\author{
J. Souchay ${ }^{1}$ and S. Bouquillon ${ }^{2}$ \\ 1 Observatoire de Paris, SYRTE, UMR 8630 du CNRS, 61 avenue de l'Observatoire, 75014 Paris, France \\ e-mail: Jean. Souchay@obspm.fr \\ 2 Tokyo National Astronomical Observatory, Oosawa 2-21-2 Mitaka Shi, Tokyo 181-0005, Japan \\ e-mail: Sebastien.Bouquillon@obspm.fr
}

Received 1 December 2003 / Accepted 22 November 2004

\begin{abstract}
We use very precise information concerning the physical properties of the asteroid Eros 433, obtained by the NEAR probe mission, to analytically compute the modifications of its rotation on a short time scale, with a precision never reached before for an asteroid. Two kinds of components are investigated here: the free rotation and the forced one, due to the gravitational torque exerted by the Sun. Coefficients of the short periodic part of Eros' forced nutation are rather large, due to the fact that the asteroid has a significant triaxial shape. They are calculated with the same relative accuracy as the moments of inertia of the asteroid. Our results are compared with those obtained through numerical integration (Miller et al. 2002, Icarus, 155, 3), which show high frequency oscillations of the position of Eros' axis of figure in space. Some important differences are discussed.
\end{abstract}

Key words. minor planets, asteroids - celestial mechanics - astrometry

\section{Introduction}

Using the very accurate database coming from the NEAR probe mission (Miller et al. 2002; Konopliv et al. 2002) we investigate here the changes of the rotation of the asteroid Eros 433 on a short time scale. As a first step, the two components of the rotation, i.e. the free motion and the forced one, are studied independantly. Then we combine them. The forced motion is due to the gravitational torque exerted principally by the Sun (we neglect in this paper the influence of the planets) and its amplitude depends strongly on the triaxial shape of the asteroid. Its main component, the nutation, i.e. the small oscillations of the axis of figure with respect to an inertial reference frame, can be modelled very accurately, both for the amplitudes and the frequencies of the series that characterize it. By contrast, the free motion depends directly on a free parameter which is the amplitude of the polar motion, that is to say the relative motion of the axis of rotation (or the axis of angular momentum) with respect to the axis of figure. This last axis is rigidly tied to the asteroid, and it corresponds classically to the axis of maximum moment of inertia. Unfortunately, the amplitude of Eros' polar motion is unknown. It is presumably very small, as is the case on Earth where its amplitude, of the order of a few 0.1" is measured daily with a sub-milliarcsecond accuracy, and on Mars, where it has never been detected. Miller et al. (2002) have proposed a value of 0'036 for the amplitude of Eros' free precession, from the data obtained by the NEAR spacecraft, and in our following calculations we choose this value as a reference for the polar motion itself. The reason is that the amplitude of the free precession in space is the same as that of the polar motion phenomena with respect to a body-tied reference frame. As a consequence, if the amplitude of the polar motion cancels, then it is also the case of the free precession.

Precise modeling of Eros'forced rotation, generated by the gravitational action of the Sun, can be obtained with precision once two kinds of data are themselves known accurately. The first one concerns the orbital elements of the asteroid. The second one concerns its physical parameters, that is to say its mass, its moments of inertia, its angular speed of rotation, and the orientation of its axis of figure with respect to space at a given date. On the other hand, the analytical modeling of free motion requires only the second kind of data.

In Table 1, we present the various parameters needed to carry out our calculations in this paper. They are taken from Miller et al. (2002), Souchay et al. (2003a), and from the MPCORB database for minor planets. The great advantage of Eros with respect to other asteroids is the relatively high accuracy of these parameters, due to the long time span during which the NEAR probe could orbit around Eros. Notice for instance that the speed of rotation has been determined with a 10 digit precision, whereas the moments of inertia $A, B$ and $C$ (in increasing order), which are fundamental to our calculations, are obtained with a 4 digit precision (Miller et al. 2002). A remarkable fact is the very large value of the mean obliquity, $\varepsilon_{0}=89^{\circ} .008$ (Souchay et al. 2003a), very close to $90^{\circ}$. As a consequence, Eros' axis of figure and axis of rotation are almost aligned along the orbital plane in a type of configuration similar to that of Uranus. 
Table 1. Eros physical and orbital data obtained from Miller et al. (2002), Souchay et al. (2003a) and from the MPCORB.

\begin{tabular}{l}
\hline \hline $\begin{array}{l}\text { Physical data obtained from Miller et al. (2002) } \\
\text { and Souchay et al. (2003a) }\end{array}$ \\
\hline Mass: $6.6904 \pm 0.003 \times 10^{15} \mathrm{~kg}$ \\
Volume: $2503 \pm 25 \mathrm{~km}^{3}$ \\
Mass per volume unit: $2.67 \mathrm{~g} / \mathrm{cm}^{3}$ \\
Large axis $a=34.6 \mathrm{~km}$ \\
Middle axis $b=19.3 \mathrm{~km}$ \\
Small axis $c=16.3 \mathrm{~km}$ \\
Moments of inertia $I_{x x}=A$ (normalized): $17.09 \mathrm{~km}^{2}$ \\
Moments of inertia $I_{y y}=B$ (normalized): $71.79 \mathrm{~km}^{2}$ \\
Moments of inertia $I_{z z}=C$ (normalized): $74.49 \mathrm{~km}^{2}$ \\
Dynamical ellipticity: $H_{\mathrm{d}}=\frac{2 C-(A+B)}{2 C}=0.40341$ \\
Rotation period: $5.27025547 \mathrm{~h}$ \\
\hline Orbital data obtained from MPCORB \\
\hline Reference epoch $t_{0}:$ June 5 th, 2002 \\
Mean anomaly at $t_{0}: 176.51285$ \\
Argument of perihelion at $t_{0}: 178.64487$ \\
$\Omega$ at $t_{0}: 304^{\circ} .40542$ \\
$i=10.83019$ \\
$e=0.2228487$ \\
$n=\dot{M}=3.5677539 \mathrm{rd} / \mathrm{y}$ \\
$a=1.4583145$ UA \\
\hline
\end{tabular}

\section{Eros' free motion}

The analytical solution for the free rotational motion of a celestial body has been studied extensively in the framework of Hamiltonian dynamics by Kinoshita $(1972,1991)$. It has been applied in the case of Eros by Souchay et al. (2003a) in the framework of a general comparison between the free motion of the Earth, Mars and Eros. In this last study, results obtained by numerical integration were compared with analytical modeling, showing a quasi perfect agreement. In parallel, the correspondence between the solutions given with the intermediary of two different sets of variables, i.e. Andoyer canonical variables $(l, g, h)$ which give the positioning of the axis of angular momentum, and Euler's angles $(\psi, \theta$ and $\phi)$ which give the positioning of the axis of figure, was checked by using Euler's kinematical equations. An agreement was reached at the level of a relative $10^{-10}$. The highly elliptical aspect of Eros' polar motion (or polhodie) was shown, which is due both to the near equality of the moments $B$ and $C$, and a large difference in $A$ from $B$ or $C$. One of the more fundamental results is the precise determination of the linear trend of the Andoyer variables $l$ and $g$ (Kinoshita 1991): the first one, $n_{\tilde{l}}=-10.16937 \mathrm{rd} / \mathrm{d}$ is the frequency of Euler's motion, that is to say the polhodie of Eros' angular momentum axis when considering the asteroid as a rigid body, the minus sign indicating that this motion is retrograde. $n_{\tilde{g}}=38.782104 \mathrm{rd} / \mathrm{d}$ is defined such that $n_{\tilde{l}}+n_{\tilde{g}}$ represents exacltly the frequency $\dot{\omega}=28.612732 \mathrm{rd} / \mathrm{d}$ of Eros' proper rotation, which corresponds to a complete rotation in

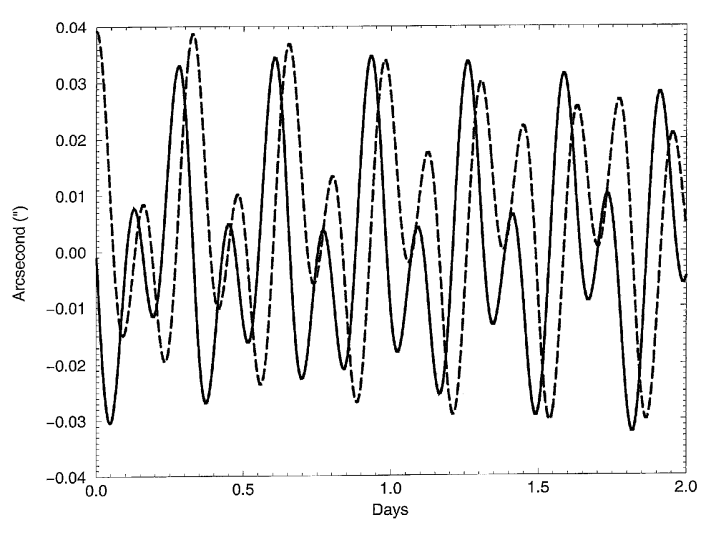

Fig. 1. Curve of short periodic Eros nutation $\Delta \psi_{\text {free }}^{\mathrm{f}}$ (in full line) and $\Delta \varepsilon_{\text {free }}^{\mathrm{f}}$ (dashed line) when considering an amplitude of 0.036 for the polar motion.

$5 \mathrm{~h} 16 \mathrm{mn} 13 \mathrm{~s}$, as was determined from the NEAR probe observations (Miller et al. 2002).

At first we concentrate on the motion of Eros' axis of figure with respect to space. Then we calculate the oscillations of the two angles $\Delta \psi_{\text {free }}^{\mathrm{f}}$ and $\Delta \varepsilon_{\text {free }}^{\mathrm{f}}$ which represent the free nutations of this axis respectively in longitude and in obliquity. Notice that in the case of a free motion, the terminology of free nutation which implies oscillations around a position of equilibrium seems more appropriate than the terminology of free precession used by several authors, such as Miller et al. (2002). Indeed, precession often refers to linear trends in longitude due to the forced rotational motion.

In the case of Eros, the values of the moments of inertia lead to a very large value of the parameter $e=\frac{\frac{1}{2}(1 / B-1 / A)}{1 / C-\frac{1}{2}(1 / A+1 / B)}$ which depends on the triaxial shape of the asteroid: here, $e=0.97785$. Then we follow exactly the same type of numerical integration as that described in detail in Souchay et al. (2003a).

\section{Motions of the axis of figure in space}

Figure 1 gives the curves described both by $\Delta \psi_{\text {free }}^{\mathrm{f}}$ and $\Delta \varepsilon_{\text {free }}^{\mathrm{f}}$ with respect to time. We notice that in both cases the signal is clearly dominated by an oscillation with a frequency of roughly 6 cycles/d and whose amplitude varies significantly, certainly due to the combination with a secondary oscillation.

In order to interpret this behaviour analytically, we denote $\psi_{\text {free }}^{\mathrm{f}}$ and $\psi_{\text {free }}^{\mathrm{a} . \mathrm{m} .}$ the precession angle in longitude respectively for the axis of figure and the axis of angular momentum. In a similar manner, we denote $\varepsilon_{\text {free }}^{\mathrm{f}}$ and $\varepsilon_{\text {free }}^{\mathrm{a} . \mathrm{m} .}$ the corresponding angles of obliquity of these two axes.

These angles are related by the classical kinematical equations (Kinoshita 1977)

$$
\begin{aligned}
& \psi_{\text {free }}^{\mathrm{f}}=\psi_{\text {free }}^{\mathrm{a} . \mathrm{m} .}+\frac{J}{\sin I} \sin g \\
& \varepsilon_{\text {free }}^{\mathrm{f}}=\varepsilon_{\text {free }}^{\mathrm{a} . \mathrm{m} .}+J \cos g+O\left(J^{2}\right) .
\end{aligned}
$$

$J$ is the angle, supposed to be very small, between the axis of figure and the axis of angular momentum, and $g$ is defined as the angle between two points (Kinoshita 1977): the first one is 
the node $N$ between the plane perpendicular to the angular momentum axis $\left(E q_{\text {a.m. }}\right)$, and the equatorial plane, perpendicular to the figure axis. The other one is the node $\gamma$ between the plane $\left(E q_{\text {a.m. }}\right)$ and a fixed plane, namely the plane of Eros'orbit at a given epoch (J2000.0). $\psi_{\text {free }}^{\text {a.m. }}$ is the angle of precession, indicating the displacement of $\left(E q_{\text {a.m. }}\right)$ along the fixed Eros orbital plane.

As we only consider free motion here, no external torque is supposed to be exerted on Eros, the angular momentum vector is constant and so we can assert that $: \psi_{\text {free }}^{\mathrm{a} . \mathrm{m}}=$ cte $=0$ and $\varepsilon_{\text {free }}^{\mathrm{f}}=$ cte $=\varepsilon_{0}$.

Therefore after derivation of the Eqs. (1) and (2) we can write:

$$
\begin{aligned}
\frac{\mathrm{d} \psi}{\mathrm{d} t} & =\frac{1}{\sin I_{0}}\left(\frac{\mathrm{d} J}{\mathrm{~d} t} \sin g+J \cos g \frac{\mathrm{d} g}{\mathrm{~d} t}\right) \\
\frac{\mathrm{d} \varepsilon}{\mathrm{d} t} & =\left(\frac{\mathrm{d} J}{\mathrm{~d} t} \cos g-J \sin g \frac{\mathrm{d} g}{\mathrm{~d} t}\right) .
\end{aligned}
$$

Kinoshita (1992) gave an exhaustive study, with the help of Hamiltonian theory and canonical variables, of the analytical expansions of torque-free rotational motions for long and short axis modes.

Folgueira and Souchay (2005) extended this last work, studying the polhodie (or polar motion) for an axisymmetric and elastic body (not a rigid one) in the Hamiltonian formalism. They modified slightly Kinoshita's expressions of the derivatives $\mathrm{d} J / \mathrm{d} t$ and $\mathrm{d} g / \mathrm{d} t$, by using other parameters, i.e. $\bar{A}$ and $\bar{\rho}$ which have the following meanings:

$\bar{A}=\frac{A+B}{2} \quad \bar{\rho}=\frac{B-A}{A+B}$.

When considering Eros as a rigid body, which seems to be acceptable to a first approximation, these expressions become simple and they can be written as follows:

$$
\begin{aligned}
& \frac{\mathrm{d} J}{\mathrm{~d} t}=\frac{\bar{\rho} G}{\bar{A}} \sin J \sin 2 l \\
& \frac{\mathrm{d} g}{\mathrm{~d} t}=\frac{G}{\bar{A}}-\frac{\bar{\rho} G}{\bar{A}} \cos 2 l
\end{aligned}
$$

where $l$ is the angle defining the polar motion of the axis of angular momentum in polar coordinates (Kinoshita 1977), $G$ is the amplitude of the angular momentum set as a constant in the present case.

By combining these expressions with (3) and (4) and taking into account the fact that $\sin J \approx J, J$ being very small, we finally get:

$\frac{\mathrm{d} \psi}{\mathrm{d} t} \approx \frac{J \omega^{\prime}}{\sin I_{0}}[\cos g-\bar{\rho} \cos (2 l+g)]$

and:

$$
\frac{\mathrm{d} \varepsilon}{\mathrm{d} t} \approx J \omega^{\prime}[-\sin g++\bar{\rho} \sin (2 l+g)]
$$

with: $\omega^{\prime}=G / \bar{A}$. And after integration of these last equations:

$$
\Delta \psi_{\text {free }}^{\mathrm{f}} \approx \frac{J \omega^{\prime}}{\sin I_{0}}\left[\frac{\sin g}{\dot{g}}-\bar{\rho} \frac{\sin (2 l+g)}{(2 \dot{l}+\dot{g})}\right]
$$

and:

$$
\Delta \varepsilon_{\text {free }}^{\mathrm{f}} \approx J \omega^{\prime}\left[\frac{\cos g}{\dot{g}}-\bar{\rho} \frac{\cos (2 l+g)}{(2 \dot{l}+\dot{g})}\right] .
$$

These expressions could have been found directly by applying analytical formulae using the variables $\tilde{l}$ and $\tilde{g}$ and the constant $e$ of Kinoshita (1992). After use of relationships between Andoyer variables, related to the angular momentum axis, and Euler angles, related to the figure axis (Kinoshita 1977), these formulae should lead to the following expressions, equivalent to (9) and (10):

$$
\Delta \psi_{\text {free }}^{\mathrm{f}} \approx \frac{\tilde{J}}{\sin I_{0}}\left[\sin \tilde{g}+\frac{1}{2} e \sin (2 \tilde{l}+\tilde{g})\right]
$$

and:

$\Delta \varepsilon_{\text {free }}^{\mathrm{f}} \approx \frac{\tilde{J}}{\sin I_{0}}\left[\cos \tilde{g}+\frac{1}{2} e \sin (2 \tilde{l}+\tilde{g})\right]$.

These expressions explain why at the first order, $\Delta \psi_{\text {free }}^{\mathrm{f}}$ and $\Delta \varepsilon_{\text {free }}^{\mathrm{f}}$ are a combination of two waves with frequency respectively $\dot{g}$ and $2 \dot{l}+\dot{g}$, thus leading to the features of the curve shown in Fig. 1. These frequencies correspond precisely to 6.1724 cycles/d and 2.9354 cycles/d respectively. The ratio of the second wave with respect to the first one is $\bar{\rho} \frac{\dot{g}}{2 \dot{+}+\dot{g}}$. With the values of $\dot{l}=n_{\tilde{l}}$ and $\dot{g}=n_{\tilde{g}}$ given at the beginning of this section, and with the values of $A$ and $B$ shown in Table 1, we get $\bar{\rho}=0.61543$ and we have a 1.2941 ratio between the amplitudes of the two waves.

\section{Polar motion and variations of the speed of rotation}

$\left(\omega_{1}, \omega_{2}, \omega_{3}\right)$ being the coordinates of Eros' rotation vector with respect to a body-fixed frame, the polar motion as well as the variations of the angular speed of rotation of Eros caused by its non axi-symmetrical shape,can be expressed in a simple way when the condition $J \ll 1$, which is equivalent to $\omega_{1} \ll \omega_{3}$ and $\omega_{2} \ll \omega_{3}$ is satisfied, as is shown by Souchay et al. (2003b). In this case the angular speed of rotation $\omega$ is quasi equivalent to $\omega_{3}$, and is subject to the following variations:

$\omega \approx \omega_{3} \approx \omega_{3}^{0}+\frac{\gamma \beta}{4 \sqrt{\lambda \mu} \omega_{3}^{0}}\left(\frac{B-A}{C}\right) \cos \left(2 \sqrt{\lambda \mu} \omega_{3}^{0}\right) t$

where $\omega_{3}^{0}$ is a constant term, and where $\lambda$ and $\mu$ are coefficients dependent on the moments of inertia:

$\lambda=\frac{C-B}{A}$ and $\mu=\frac{C-A}{B}$,

here, $\gamma$ and $\beta$ are the semi-major and the semi-minor axis of the quasi-elliptical polar motion of the axis of rotation, i.e., the relative motion of the axis of rotation with respect to the axis of figure:

$\omega_{1}=\beta \sin \left(\sqrt{\lambda \mu} \omega_{3}^{0} t\right) \quad \omega_{2}=\gamma \cos \left(\sqrt{\lambda \mu} \omega_{3}^{0} t\right)$.

Notice that $\gamma$ and $\beta$ are dependent parameters:

$\gamma=-\sqrt{\frac{\mu}{\lambda}} \times \beta$ 
Moreover, the period of Eros' polar motion is given by the relationships:

$$
T=\frac{2 \pi}{\sqrt{\lambda \mu} \omega_{3}^{0}} \text {. }
$$

Notice that this expression is the same as that found by Black et al. (1999) who studied a possible rotation state of Eros.

The only parameter which remains to be determined is the amplitude $\beta$ (or $\gamma$ ) of the polar motion, which is a free parameter, and whose value is very close to $J$, the axis of angular momentum and axis of rotation being very close to each other.

According to a previous remark, and as can be shown by Eqs. (9) and (10), the amplitude of $J$ is roughly the same as the amplitude of the free nutation (or free precession according to the following authors), which was presumed to be 0.036 by Miller et al. (2002). We chose this same value for the amplitude of $J$ in the equations above.

By using the physical characteristics of Eros from Table 1, we find that the eccentricity $e_{\mathrm{pm}}$ of the ellipse of polar motion deduced from the ratio $\gamma / \beta$ in (16) is very high, that is to say $e_{\mathrm{pm}}=0.8952$, and the period of the polar motion, given by (17), is $0.6178 \mathrm{~d}$. In contrast to the Earth, for which the polar motion is much slower than the proper rotation (by a factor of more than 300), in the case of Eros it is of the same order, that is to say 14.6 hours. Lastly, because of the very small values of $\gamma$ and $\beta$ in the numerator of the expression on the right-hand side of Eq. (13), it can be easily shown that the variations of $\omega$ are negligible.

\section{High frequency Eros rotational variations due to the disturbing solar potential}

Eros' disturbing potential due to the Sun can be represented by expansions in spherical harmonics, in the following manner (Tisserand 1895; Kinoshita 1977):

$$
\begin{aligned}
U= & \frac{\kappa^{2} M_{\mathrm{S}}}{r^{3}} \times\left[\left(\frac{2 C-A-B}{2}\right) P_{0}^{2}(\sin \delta)\right. \\
& \left.+\left(\frac{A-B}{4}\right) P_{2}^{2}(\sin \delta) \cos 2 \alpha\right] \\
& +\sum_{n=3}^{\infty} \frac{\kappa^{2} m_{\mathrm{E}} M_{\mathrm{S}} a_{\mathrm{E}}^{n}}{r^{n+1}}\left[J_{n} P_{n}^{0}(\sin \delta)\right. \\
& \left.-\sum_{m=1}^{n} P_{n}^{m}(\sin \delta)\left(C_{n, m} \cos m \alpha+S_{n, m} \sin m \alpha\right)\right]
\end{aligned}
$$

In this formula, $P_{n}^{m}$ are Legendre polynomials, $A, B, C$ stand for Eros' principal moments of inertia, in increasing order of amplitude. $m_{\mathrm{E}}$ and $M_{\mathrm{S}}$ are respectively the mass of Eros and the mass of the Sun. $J_{n}, C_{n, m}$ and $S_{n, m}$ are the coefficients of Eros potential which have been determined with remarkable accuracy for the first time in the case of an asteroid (Miller et al. 2002). $r$ represents the distance between Eros' center of mass and the Sun, and $a_{\mathrm{E}}$ is the mean value of Eros radius. $\alpha$ and $\delta$ represent the coordinates of the perturbing body, i.e., the Sun, in a reference frame based on the Eros equator and principal axes: $\delta$ is the latitude of the Sun with respect to the
Eros equator, and $\alpha$ the longitude of the Sun counted from the prime meridian which crosses the axis of minimum moment of inertia $A$.

The influence of the first term of the potential on the right-hand side of Eq. (18), depending on Eros flattening and dynamical ellipticity $H_{\mathrm{d}}=\frac{2 C-A-B}{2 C}$ has been studied in detail recently (Souchay et al. 2003a). It generates long periodic nutations which reach about 55" peak to peak, with much larger amplitudes in obliquity $\Delta \varepsilon$ as in longitude $\Delta \psi$, because of the very high value of the obliquity $\left(\varepsilon \approx 89^{\circ}\right)$. The analytical developments of these nutations (Souchay et al. 2003a) have been given at the 4th order of the eccentricity, as a function of Eros mean motion $M$. The rate of precession has been fixed at the value 2 '. $84 / y$.

In this paper, we study the specific influence of Eros' triaxiality on the potential exerted by the Sun, which is represented by the second term on the right hand side of Eq. (18), depending on the coefficient $(A-B) / 4$. The terms at higher order of the potential, with $n \gg 3$, which also generate very short periodic oscillations of the axis of figure, can be totally neglected because of the scaling factor $\left(\frac{a_{\mathrm{E}}}{r}\right)^{(n-2)}$ with respect to leading terms depending on the flattening, $a_{\mathrm{E}}$ being roughly $5 \times 10^{-6}$ smaller than the distance $r$. As a result, we limit our study to the following part of the potential:

$U_{\text {triax }}=\frac{\kappa^{2} M_{\mathrm{S}}}{r^{3}} \times\left[\left(\frac{A-B}{4}\right) P_{2}^{2}(\sin \delta) \cos 2 \alpha\right]$.

The variations at high frequency coming from this component are due to the presence of the argument $2 \alpha$ in the potential, which is twice the sidereal angle of Eros' rotation. Considering that this rotation is accomplished in about $5.270 \mathrm{~h}$ (Miller et al. 2002), and considering that $P_{2}^{2}(\sin \delta)$ varies slowly with respect to time, its combination with $\cos 2 \alpha$ will result in Fourier's series with terms with a period close to half the period of rotation of Eros, that is to say roughly $2.635 \mathrm{~h}$.

The procedure used to calculate Eros' nutation due to the triaxiality starting from Eq. (17) and using canonical equations is fully explained by Kinoshita (1977). As we are adopting exactly the same procedure, we only summarize the principal steps here. First, the determining function associated with the triaxiality is obtained by the integration of the periodic part of $U_{\text {triax }}$ :

$W_{\text {triax }}=\frac{\kappa^{2} M_{\mathrm{S}}}{a^{3}} \times\left[\frac{A-B}{4}\right] \int\left[\left(\frac{a^{3}}{r}\right) P_{2}^{2}(\sin \delta) \cos 2 \alpha\right]_{\text {per. }} \mathrm{d} t$

$P_{2}^{2}(\sin \delta) \cos 2 \alpha$ can be expressed as a function of the coordinates $\lambda$ and $\beta$ of the perturbing body, i.e. the Sun, with respect to a given fixed plane, through the intermediary of the modified Jacobi polynomials (Kinoshita et al. 1974). $\lambda$ and $\beta$ are respectively the longitude and the latitude of the Sun. In order to follow the same kind of parametrization as for the Earth and for Mars, we choose as a reference plane Eros' orbital plane, so that the value of $\beta$ can be set to $\beta=0$, whereas $\lambda$ is determined starting from the ascending node of Eros'orbit with respect to Eros'equator which can be considered as Eros'equinox. 
We calculate the following expressions as a sum of sinusoids with coefficients $A_{v}$ and $B_{v}$, and with argument $\Theta_{v}$ :

$\frac{1}{2}\left[\frac{a}{r}\right]^{3}=\sum A_{v}^{0} \cos \Theta_{v}$

$\left[\frac{a}{r}\right]^{3} \sin \beta \cos \beta \sin \lambda=\sum A_{v}^{1} \cos \Theta_{v}=0$

$\left[\frac{a}{r}\right]^{3} \cos ^{2} \beta \cos 2 \lambda=\sum A_{v}^{2} \cos \Theta_{v}$.

Souchay et al. (2003a) have shown that the two expressions (21) and (23) above can be developed as a function of Eros' mean anomaly $M$, Eros' eccentricity $e$ and of two terms $C$ and $S$ which can be taken as constants for a relatively long time span. The reason is that they involve only long periodic motions. The definitions of $C$ and $S$ are given by Souchay et al. (2003a). Their numerical values at J2000.0 are: $C=0.85777$ and $S=0.51409$.

In Tables 2 and 3 we gather the analytical expressions respectively of the coefficients $A_{v}^{0}$ and $A_{v}^{2}$ as a function of the constant terms $C$ and $S$, together with their numerical value for J2000.0 and the corresponding $\Theta_{v}$ expressed as a function of Eros'mean anomaly $M$.

Then, the determining function $W_{\text {triax }}$ of Eq. (18) can be transformed in the following way (Kinoshita 1977):

$$
\begin{aligned}
W_{\text {triax }}= & \frac{3}{2} \sin ^{2} J W_{\text {triax }}^{0}+\sum_{\rho= \pm 1} \sin J(1+\rho \cos J) W_{\text {triax }}^{1} \\
& +\frac{1}{4} \sum_{\rho= \pm 1}(1+\rho \cos J)^{2} W_{\text {triax }}^{2} .
\end{aligned}
$$

With the following expressions:

$$
\begin{aligned}
W_{\text {triax }}^{0}= & \frac{3 \kappa^{2} M_{\mathrm{S}}(B-A)}{2 a^{3}} \sum_{v} \sum_{\mu= \pm 1} \frac{B_{v}}{2 n_{l}-\mu N_{v}} \\
& \times \sin \left(2 \rho \mu \Theta_{v}\right) \\
W_{\text {triax }}^{1}= & \frac{3 \kappa^{2} M_{\mathrm{S}}(B-A)}{2 a^{3}} \\
& \times \sum_{v} \sum_{\mu= \pm 1} \frac{C_{v}(\mu)}{n_{g}+2 \rho n_{l}-\mu N_{v}} \sin \left(g+2 \rho l-\mu \Theta_{v}\right) \\
W_{\text {triax }}^{2}= & \frac{3 \kappa^{2} M_{\mathrm{S}}(B-A)}{2 a^{3}} \\
& \times \sum_{v} \sum_{\mu= \pm 1} \frac{D_{v}(\mu)}{2 n_{g}+2 \rho n_{l}-\mu N_{v}} \sin \left(2 g+2 \rho l-\mu \Theta_{v}\right) .
\end{aligned}
$$

We have the following notations (Kinoshita 1977), taking into account the fact that the coefficients $A_{v}^{1}$ are equal to 0 :

$$
\begin{aligned}
B_{v} & =-\frac{1}{6}\left(3 \cos ^{2} \varepsilon_{0}-1\right) A_{v}^{0}-\frac{1}{4} \sin ^{2} \varepsilon_{0} A_{v}^{2} \\
C_{v}(\mu) & =\frac{1}{4} \sin \varepsilon_{0} A_{v}^{0}-\frac{1}{4} \mu \sin \varepsilon_{0}\left(1+\mu \cos \varepsilon_{0}\right) A_{v}^{2} \\
D_{v}(\mu) & =-\frac{1}{2} \sin ^{2} \varepsilon_{0} A_{v}^{0}-\frac{1}{4}\left(1+\mu \cos \varepsilon_{0}\right)^{2} A_{v}^{2} .
\end{aligned}
$$

Recall that the angle $J$ stands for the angular offset between the
Table 2. Expression and numerical value of the coefficients $A_{v}^{0}$ in Eqs. (19)-(21) together with the corresponding angle $\Theta_{v}$.

\begin{tabular}{lrr}
\hline \hline Angle $\Theta_{v}$ & Expression of the coefficient $A_{v}^{0}$ & Value \\
\hline 0 & $\frac{1}{2}+\frac{3}{4} e^{2}+\frac{15}{16} e^{4}$ & 0.53955828 \\
$M$ & $\frac{3}{2} e+\frac{27}{16} e^{3}$ & 0.35294863 \\
$2 M$ & $\frac{9}{4} e^{2}+\frac{7}{4} e^{4}$ & 0.11605444 \\
$3 M$ & $\frac{53}{16} e^{3}$ & 0.03665947 \\
$4 M$ & $\frac{77}{16} e^{4}$ & 0.01186891 \\
\hline
\end{tabular}

Table 3. Expression and numerical value of the coefficients $A_{v}^{2}$ in Eq. (21) together with the corresponding angle $\Theta_{v}$.

\begin{tabular}{lrr}
\hline \hline Angle $\Theta_{v}$ & Expression of the coefficient $A_{v}^{2}$ & Value \\
\hline$M$ & $C\left[-\frac{e}{2}+\frac{e^{3}}{12}\right]$ & -0.09447812 \\
$M-\frac{\pi}{2}$ & $S\left[\frac{e}{2}-\frac{e^{3}}{24}\right]$ & 0.05704579 \\
$2 M$ & $C\left[1-\frac{5}{2} e^{2}+\frac{41}{4} e^{4}\right]$ & 0.75304852 \\
$2 M-\frac{\pi}{2}$ & $S\left[-1+\frac{5}{2} e^{2}-\frac{37}{48} e^{4}\right]$ & -0.45124669 \\
$3 M$ & $C\left[\frac{7 e}{2}-\frac{123}{16} e^{4}\right]$ & 0.59603207 \\
$3 M-\frac{\pi}{2}$ & $C\left[-\frac{7 e}{2}+\frac{123}{16} e^{4}\right]$ & -0.35724190 \\
$4 M$ & $C\left[\frac{17}{2} e^{2}-\frac{115}{6} e^{4}\right]$ & 0.32152353 \\
$4 M-\frac{\pi}{2}$ & $S\left[-\frac{17}{2} e^{2}+\frac{115}{6} e^{4}\right]$ & -0.19271056 \\
$5 M$ & $C \frac{845}{48} e^{4}$ & 0.16710813 \\
$5 M-\frac{\pi}{2}$ & $-S \frac{845}{48} e^{4}$ & -0.10015908 \\
$6 M$ & $C \frac{533}{16} e^{4}$ & 0.07046922 \\
$6 M-\frac{\pi}{2}$ & $-S \frac{845}{48} e^{4}$ & -0.04223692 \\
\hline
\end{tabular}

axis of figure and the axis of angular momentum. As it is the case for the Earth, where this angle does not exceed 1", as well as for the other planets, we can assume that this angle is very close to 0 . We have explained above that it roughly corresponds to the amplitude of Eros' free precession in space which might be 0 '036 (Miller et al. 2002). Therefore the terms containing $\sin J$ and $\sin ^{2} J$ in $(22)$ can be neglected, and $\cos J \approx 1$. So, to a first approximation, $W_{\text {triax }}$ can be noticeably simplified and rewritten in the following way:

$$
\begin{aligned}
W_{\text {triax }} \approx & W_{\text {triax }}^{2} \approx \frac{3 \kappa^{2} M_{\mathrm{S}}}{2 a^{3}}(B-A) \\
& \times \sum_{\nu} \sum_{\mu= \pm 1} \frac{D_{v}(\mu)}{2 n_{g}+2 n_{l}-\mu N_{v}} \sin \left(2 g+2 l-\mu \Theta_{\nu}\right)
\end{aligned}
$$

which can be also written as:

$$
\begin{aligned}
W_{\text {triax }} \approx & \frac{3 \kappa^{2} M_{\mathrm{S}}}{2 a^{3}}(B-A) \\
& \times \sum_{v} \sum_{\mu= \pm 1}\left[-\frac{1}{2} \sin ^{2} \varepsilon_{0} A_{v}^{0}-\frac{1}{4}\left(1+\mu \cos \varepsilon_{0}\right)^{2} A_{v}^{2}\right] \\
& \times \frac{\sin \left(2 g+2 l-\mu \Theta_{v}\right)}{2 n_{l}+2 n_{g}-\mu N_{v}}
\end{aligned}
$$


By applying canonical equations, we find the expressions of the nutation of Eros' axis of angular momentum (Kinoshita 1977):

$$
\begin{aligned}
{[\Delta \psi]_{\text {forced }}^{\text {a.m. }}=} & -\frac{B-A}{2 C-A-B} \frac{k}{\sin \varepsilon_{0}} \\
& \times \sum_{v} \sum_{\mu= \pm 1} \frac{C_{v}(\mu)}{2 n_{l}+2 n_{g}-\mu N_{v}} \sin \left(2 g+2 l-\mu \Theta_{v}\right) \\
= & \frac{B-A}{2 C-A-B} \frac{k}{\sin \varepsilon_{0}} \\
& \times \sum_{v} \sum_{\mu= \pm 1}\left[-\frac{1}{4} \sin 2 \varepsilon_{0} A_{v}^{0}+\frac{1}{4} \mu \sin \varepsilon_{0}\left(1+\mu \cos \varepsilon_{0}\right) A_{v}^{2}\right] \\
& \times \frac{\sin \left(2 g+2 l-\mu \Theta_{v}\right)}{2 n_{l}+2 n_{g}-\mu N_{v}}
\end{aligned}
$$

and:

$$
\begin{aligned}
{[\Delta \varepsilon]_{\text {forced }}^{\text {a.m. }}=} & -\frac{B-A}{2 C-A-B} k \sum_{v} \sum_{\mu= \pm 1} \frac{C_{v}(\mu)}{2 n_{l}+2 n_{g}-\mu N_{v}} \\
& \times \cos \left(2 g+2 l-\mu \Theta_{v}\right) \\
= & \frac{B-A}{2 C-A-B} k \\
& \times \sum_{v} \sum_{\mu= \pm 1}\left[-\frac{1}{4} \sin 2 \varepsilon_{0} A_{v}^{0}+\frac{1}{4} \mu \sin \varepsilon_{0}\left(1+\cos \varepsilon_{0}\right) A_{v}^{2}\right] \\
& \times \frac{\cos \left(2 g+2 l-\mu \Theta_{v}\right)}{2 n_{l}+2 n_{g}-\mu N_{v}} .
\end{aligned}
$$

Then we can calculate the high frequency nutations of the axis of figure due to the triaxiality with the help of the kinematic formula linking the position angles of the figure axis $\psi^{\mathrm{f}}$ and $\varepsilon^{\mathrm{f}}$ to the respective positions of the angular momentum axis $\psi^{\text {A.M. }}$ and $\varepsilon^{\text {A.M. }}$, with the intermediary of the Andoyer variables:

$$
\begin{aligned}
\psi^{\mathrm{f}}-\psi^{\mathrm{a} . \mathrm{m}} . & =\frac{J}{\sin \varepsilon_{0}} \sin g \\
\varepsilon^{\mathrm{f}}-\varepsilon^{\text {a.m. }} & =-J \cos g .
\end{aligned}
$$

Notice that the displacements of Eros' axis of figure have been calculated by Souchay et al. (2003a) with respect to those of the axis of angular momentum using same formula, by the intermediary of what is classically named Oppolzer terms. Now, we can link the nutations of the two axes:

$$
\begin{aligned}
\Delta \psi_{\text {forced }}^{\mathrm{f}} & =\Delta \psi_{\text {forced }}^{\text {a.m. }}+\Delta\left(\frac{J}{\sin \varepsilon_{0}} \sin g\right) \\
\Delta \varepsilon_{\text {forced }}^{\mathrm{f}} & =\Delta \varepsilon_{\text {forced }}^{\text {a.m. }}-\Delta(J \cos g) .
\end{aligned}
$$

We still get the expressions of $\Delta(J \sin g)$ and $\Delta(J \cos g)$ from Kinoshita (1977). They lead to the final formulations for the nutation of the figure axis:

$$
\begin{aligned}
& {[\Delta \psi]_{\text {forced }}^{\mathrm{f}}=\frac{B-A}{2 C-A-B} \frac{k}{\sin \varepsilon_{0}}} \\
& \quad \times \sum_{v} \sum_{\mu= \pm 1}\left[-\frac{1}{4} \sin 2 \varepsilon_{0} A_{v}^{0}+\frac{1}{4} \mu \sin \varepsilon_{0}\left(1+\mu \cos \varepsilon_{0}\right) A_{v}^{2}\right] \\
& \quad \times\left[\frac{1}{2 n_{l}+2 n_{g}-\mu N_{v}}-\frac{1}{2 n_{l}+2 n_{g}-\mu N_{v}}\right] \sin \left(2 g+2 l-\mu \Theta_{v}\right)
\end{aligned}
$$

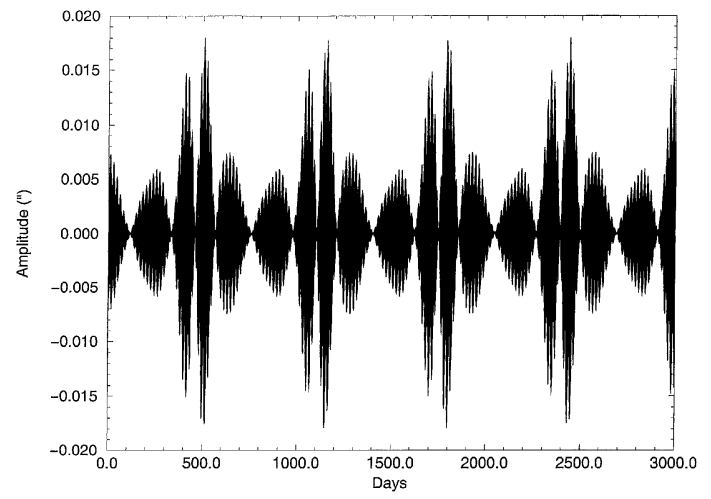

Fig. 2. Curve of Eros forced nutation $\Delta \psi_{\text {triax }}^{\mathrm{f}}$ due to the gravitational torque exerted by the Sun on the triaxial shape of the asteroid.

$$
\begin{aligned}
& {[\Delta \varepsilon]_{\text {forced }}^{\mathrm{f}}=\frac{B-A}{2 C-A-B} k} \\
& \quad \times \sum_{v} \sum_{\mu= \pm 1}\left[-\frac{1}{4} \sin 2 \varepsilon_{0} A_{v}^{0}+\frac{1}{4} \mu \sin \varepsilon_{0}\left(1+\mu \cos \varepsilon_{0}\right) A_{v}^{2}\right] \\
& \quad \times\left[\frac{1}{2 n_{l}+2 n_{g}-\mu N_{v}}-\frac{1}{2 n_{l}+2 n_{g}-\mu N_{v}}\right] \cos \left(2 g+2 l-\mu \Theta_{v}\right) .
\end{aligned}
$$

In Tables 4 and 5 we gather the coefficients of the high frequency nutations $\Delta \psi_{\text {forced }}^{\mathrm{f}}$ and $\Delta \varepsilon_{\text {forced }}^{\mathrm{f}}$, due to the triaxial shape of the asteroid. As we have explained previously, the period of each sinusoidal term is very closed to $2^{\mathrm{h}} 38^{\mathrm{mn}} 06^{\mathrm{s}}$, which corresponds to half the period of Eros'rotation. Therefore the curve of the total amount for both nutations, shown for $\Delta \psi_{\text {forced }}^{\mathrm{f}}$ in Fig. 2, is a beating characterized by large variations of the maximum amplitude, sometimes cancelling, sometimes reaching a 0.035 peak-to-peak amplitude. Notice that the amplitude undergoes variations with a very stable and characteristic feature according to a cycle of roughly 650 days. The curve for $\Delta \varepsilon_{\text {forced }}^{\mathrm{f}}$ is quite similar, because the equations for $\Delta \psi_{\text {forced }}^{\mathrm{f}}$ and $\Delta \varepsilon_{\text {forced }}^{\mathrm{f}}$ differ only by a scaling factor $\sin \varepsilon_{0}$ very close to $1\left(\varepsilon_{0}=89^{\circ} .008\right)$, and a phase equal to $\pi / 2$. Thus $\Delta \varepsilon_{\text {forced }}^{\mathrm{f}}$ is slightly shifted with respect to $\Delta \psi_{\text {forced }}^{\mathrm{f}}$ (a $\pi / 2$ phase of the proper rotation corresponds to roughly a $1.3 \mathrm{~h}$ shift).

\section{Combined effects of free and forced nutations}

In the two previous sections, we calculated the short periodic parts both of Eros' free nutation $\left(\Delta \psi_{\text {free }}^{\mathrm{f}}, \Delta \varepsilon_{\text {free }}^{\mathrm{f}}\right)$ and forced nutation $\left(\Delta \psi_{\text {forced }}^{\mathrm{f}}, \Delta \varepsilon_{\text {forced }}^{f}\right)$ due to the gravitational forcing exerted by the Sun. Below, we shall investigate the general features of the path described by Eros' pole of figure with respect to an inertial frame.

In Fig. 3 we illustrate (on the same graphs) the variations, for $\mathrm{J} 2000.0$, of $\Delta \psi_{\text {forced }}^{\mathrm{f}}$ (in dashed line) and $\Delta \psi_{\text {free }}^{\mathrm{f}}$ (in bold and slightly dotted line). Their combination is given by the thin full line curve. Notice that we have again chosen the value of $J=0$.'036 for the maximal amplitude of the polar motion which gives the same amplitude for the free nutation, as has been already explained and shown in Fig. 1. This last value corresponds to a maximum threshold taken from Miller et al. (2002), and might be subject to variations according to time, as is the case for the Earth, due to the action of dissipative 
Table 4. Coefficients of the high-frequency forced nutation in longitude $\Delta \psi_{\text {forced }}^{\mathrm{f}}$ of Eros. $\omega$ is the argument for the sidereal rotation of Eros, and $M$ is Eros' mean anomaly.

\begin{tabular}{lrrr}
\hline \hline Angle & Sine $\left(0{ }^{\prime} 001\right)$ & Cosine $\left(00^{\prime} 001\right)$ & Period $(\mathrm{h})$ \\
\hline $2 \omega$ & 0.0846 & 0.0000 & $2^{\mathrm{h}} 38^{\mathrm{mn}} 06^{\mathrm{s}}$ \\
$2 \omega-M$ & 0.4890 & -0.2610 & $2^{\mathrm{h}} 38^{\mathrm{mn}} 08^{\mathrm{s}}$ \\
$2 \omega+M$ & -0.4182 & -0.2517 & $2^{\mathrm{h}} 38^{\mathrm{mn}} 05^{\mathrm{s}}$ \\
$2 \omega-2 M$ & -3.4287 & 2.0655 & $2^{\mathrm{h}} 38^{\mathrm{mn}} 10^{\mathrm{s}}$ \\
$2 \omega+2 M$ & 3.3211 & 1.9900 & $2^{\mathrm{h}} 38^{\mathrm{mn}} 03^{\mathrm{s}}$ \\
$2 \omega-3 M$ & -2.7241 & 1.6361 & $2^{\mathrm{h}} 38^{\mathrm{mn}} 11^{\mathrm{s}}$ \\
$2 \omega+3 M$ & 2.6271 & 1.5746 & $2^{\mathrm{h}} 38^{\mathrm{mn}} 02^{\mathrm{s}}$ \\
$2 \omega-4 M$ & -1.4715 & 0.8831 & $2^{\mathrm{h}} 38^{\mathrm{mn}} 13^{\mathrm{s}}$ \\
$2 \omega+4 M$ & 1.4636 & 0.8489 & $2^{\mathrm{h}} 38^{\mathrm{mn}} 00^{\mathrm{s}}$ \\
$2 \omega-5 M$ & -0.7662 & 0.4592 & $2^{\mathrm{h}} 38^{\mathrm{mn}} 15^{\mathrm{s}}$ \\
$2 \omega+5 M$ & 0.7357 & 0.4409 & $2^{\mathrm{h}} 37^{\mathrm{mn}} 58^{\mathrm{s}}$ \\
$2 \omega-6 M$ & -0.3236 & 0.1937 & $2^{\mathrm{h}} 38^{\mathrm{mn}} 16^{\mathrm{s}}$ \\
$2 \omega+6 M$ & 0.3100 & 0.1858 & $2^{\mathrm{h}} 37^{\mathrm{mn}} 57^{\mathrm{s}}$ \\
\hline
\end{tabular}

Table 5. Coefficients of the high-frequency forced nutation in obliquity $\Delta \varepsilon_{\text {forced }}^{\mathrm{f}}$ of Eros. $\omega$ is the argument for the sidereal Eros'rotation, and $M$ is Eros' mean anomaly.

\begin{tabular}{lrrl}
\hline \hline Angle & Sine $\left(0^{\prime} \cdot 001\right)$ & Cosine $\left(00^{\prime} 001\right)$ & Period $(\mathrm{h})$ \\
\hline $2 \omega$ & 0.0846 & 0.0000 & $2^{\mathrm{h}} 38^{\mathrm{mn}} 06^{\mathrm{s}}$ \\
$2 \omega-M$ & -0.4889 & -0.2609 & $2^{\mathrm{h}} 38^{\mathrm{mn}} 08^{\mathrm{s}}$ \\
$2 \omega+M$ & 0.4182 & -0.2517 & $2^{\mathrm{h}} 38^{\mathrm{mn}} 05^{\mathrm{s}}$ \\
$2 \omega-2 M$ & 3.4287 & 2.0655 & $2^{\mathrm{h}} 38^{\mathrm{mn}} 10^{\mathrm{s}}$ \\
$2 \omega+2 M$ & -3.3205 & 1.9900 & $2^{\mathrm{h}} 38^{\mathrm{mn}} 03^{\mathrm{s}}$ \\
$2 \omega-3 M$ & 2.7241 & 1.6361 & $2^{\mathrm{h}} 38^{\mathrm{mn}} 11^{\mathrm{s}}$ \\
$2 \omega+3 M$ & -2.6271 & 1.5746 & $2^{\mathrm{h}} 38^{\mathrm{mn}} 02^{\mathrm{s}}$ \\
$2 \omega-4 M$ & 1.4715 & 0.8831 & $2^{\mathrm{h}} 38^{\mathrm{mn}} 13^{\mathrm{s}}$ \\
$2 \omega+4 M$ & -1.4636 & 0.8489 & $2^{\mathrm{h}} 38^{\mathrm{mn}} 00^{\mathrm{s}}$ \\
$2 \omega-5 M$ & 0.4592 & -0.7357 & $2^{\mathrm{h}} 38^{\mathrm{mn}} 15^{\mathrm{s}}$ \\
$2 \omega+5 M$ & -0.7357 & 0.4409 & $2^{\mathrm{h}} 37^{\mathrm{mn}} 58^{\mathrm{s}}$ \\
$2 \omega-6 M$ & 0.3233 & 0.1937 & $2^{\mathrm{h}} 38^{\mathrm{mn}} 16^{\mathrm{s}}$ \\
$2 \omega+6 M$ & -0.3100 & 0.1858 & $2^{\mathrm{h}} 37^{\mathrm{mn}} 57^{\mathrm{s}}$ \\
\hline
\end{tabular}

internal mechanisms. We must emphasize the fact that its choice is subject to some uncertainty, as it has been identified with what was named by Miller et al. as Eros' free precession. Moreover some precisions concerning the definition of this free precession and the way in which it could be determined from the NEAR mission has not been clearly explained (Miller et al. 2002). However the amplitude of $J$ does not influence the aspect of the curve described by the figure axis with respect to space, but only its scale.

In Fig. 4 we show the two dimensional trajectories described by Eros' pole of figure when undergoing the free and forced motions separately. The first one $\left(\Delta \psi_{\text {free }}^{\mathrm{f}}, \Delta \varepsilon_{\text {free }}^{\mathrm{f}}\right)$ can be considered as a curve made of a simple loop pattern reproduced indefinitely after being shifted by rotation, whereas the second

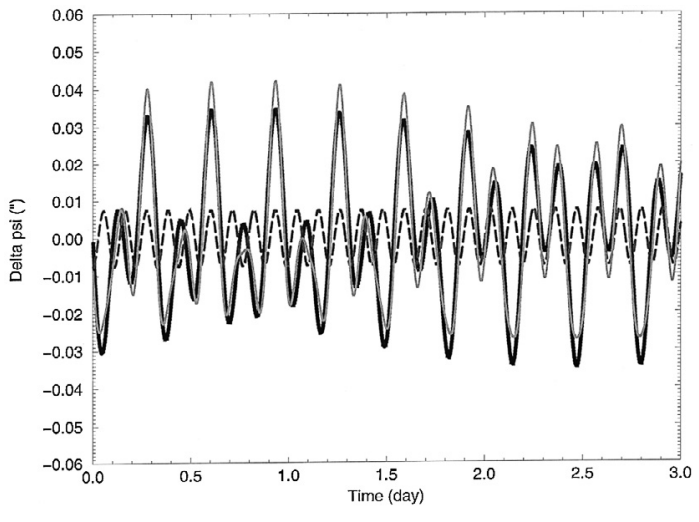

Fig. 3. Curve of the free nutation (in bold dotted line) $\Delta \psi_{\text {free }}^{\mathrm{f}}$ and the forced one $\Delta \psi_{\text {triax }}^{\mathrm{f}}$ (in dashed line). The two curves are summed (in full thin line).

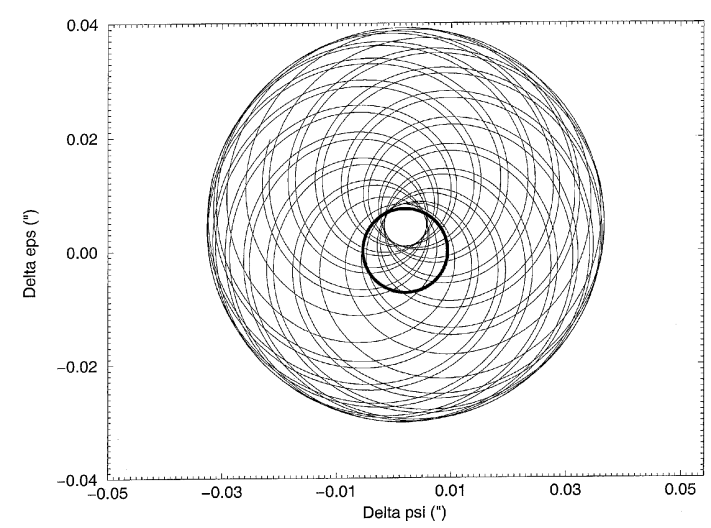

Fig. 4. Curve representing the free nutation loop of the figure axis $\Delta \varepsilon_{\text {free }}$ as a function of $\Delta \psi_{\text {triax }}^{\text {free }}$, together with the forced high fequency nutation loop (circular) $\Delta \varepsilon_{\text {triax }}^{\text {free }}$ as a function of $\Delta \psi_{\text {triax }}^{\mathrm{f}}$.

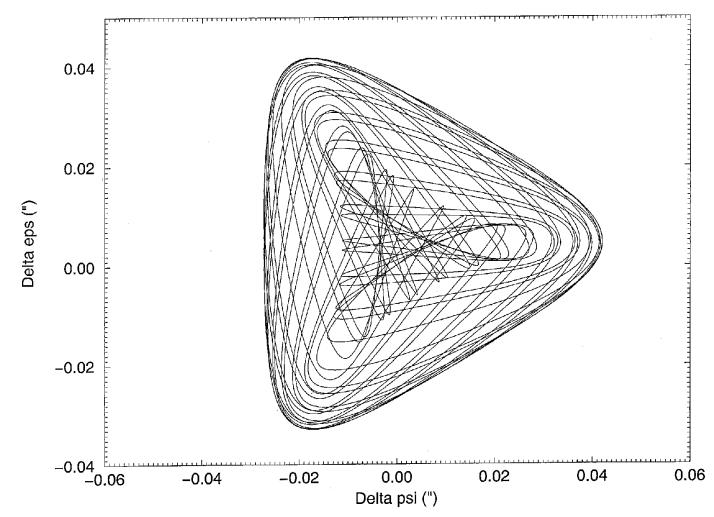

Fig. 5. Curve representing the short periodic motion of the pole of Eros' figure axis for a short time span.

one, $\left(\Delta \psi_{\text {forced }}^{\mathrm{f}}, \Delta \varepsilon_{\text {forced }}^{\mathrm{f}}\right)$ is a quasi-circular curve, with a radius varying according to time, as was shown in Fig. 2. Figures 5.1 and 5.2 show respectively for a long and a short time span the motion of the axis of figure undergoing the two motions combined. The path is included inside a triangular boundary, the amplitude of each side of the triangle being about 0 .' $^{\prime} 08$. Notice the regularity of the curve and its symmetry after rotation. 


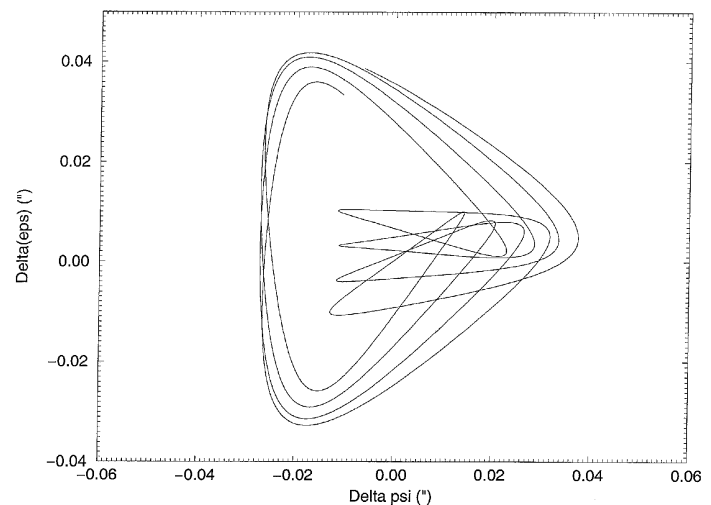

Fig. 6. Curve representing the short periodic motion of the pole of Eros' figure axis for a long time span.

The representation of Eros pole of figure in a bidimensional $\alpha, \delta$ coordinate curve, when considering all the effects of the precession, the long periodic nutations and the short periodic ones, can also be carried out once the necessary transformations from the reference frame relating to Eros to that relating to the Earth's equator and equinox have been done. These transformations were used by Souchay et al. (2003a) when studying the long periodic motions of Eros' pole. The values of the precession and of the long periodic nutations were also taken from this last paper. The curve is shown in Fig. 6: the linear trend is due to the long-periodic nutations in obliquity whose effects for a small time span are far greater than the long-periodic nutations in longitude and the precession in longitude itself, as was demonstrated analytically by Souchay et al. (2003a). The effects of the short-periodic nutations are clearly visible: they consist of loops with aspect and amplitude varying in a noticeable manner, characterized in particular by retrogradations due to the sinusoidal variations of the obliquity calculated in the preceding sections.

We can compare this last curve with that presented by Miller et al. (2002), probably obtained by numerical integration. In this last study, although high frequency oscillations of the pole exist, the high frequency loops obtained in Fig. 6 are not present: this should indicate that only the high-frequency variations of Eros' pole in longitude, not in obliquity, have been taken into account. This hypothesis is confirmed by an experimental study performed by us, consisting of only taking into account the terms of free nutation in longitude, not in obliquity: with that intentional omission the curve becomes quite similar to that of the authors above.

On the contrary, notice that Souchay et al. (2003a) found an excellent agreement between their calculations and those of Miller et al. (2002) concerning the long-periodic behaviour of the motion of Eros' pole, including both forced precession and forced long-periodic nutation.

\section{Conclusion}

As is the case for the Earth, the variations of rotation of Eros can be divided into several components: one is the displacement of the axis of figure in space, split itself in two parts, i.e. the secular motion of precession and the periodic

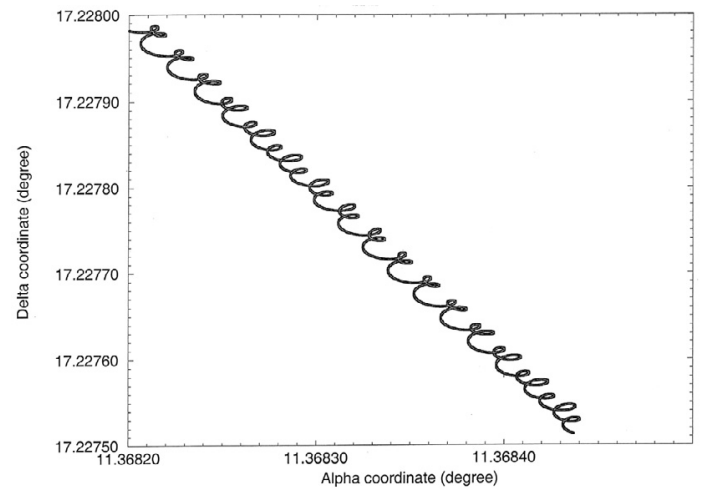

Fig. 7. Curve representing the $(\alpha, \delta)$ variations of the equatorial coordinates of Eros' figure axis, all nutations and precession contributions being included, according to this paper and Souchay et al. (2003a).

oscillations characterizing the nutation. Another component is the polar motion, or polhody, that is to say the relative motion of the axis of rotation (or possibly the axis of angular momentum), with respect to the axis of figure. Also we must mention the variations of the angular speed of rotation.

In this paper, we have achieved an exhaustive study of Eros' rotation in a fully analytical manner, thus completing a previous study (Souchay et al. 2003a) which dealt only with forced precession and forced long periodic nutations. Thanks to very accurate and high-precision data obtained from the NEAR probe orbiting around the asteroid Eros, it was possible in this paper to model with exceptional precision the high frequency components of the free rotation, as well as the forced one due to the gravitational torque exerted by the Sun.

We have shown that the free rotation generates for the axis of figure, both for $\Delta \psi_{\text {free }}^{\mathrm{f}}$ and $\Delta \varepsilon_{\text {free }}^{\mathrm{f}}$ two leading oscillations, with a frequency of 6.17 cycles/day and 2.93 cycles/day and close amplitudes. These amplitudes depend on the amplitude of the polar motion, which is a free parameter. We have chosen the value of 0 '036 (Miller et al. 2002), which gives a free precession with this same amount.

We have also computed up to the 4th digit of precision the coefficients of high-frequency nutations of the figure axis of the asteroid, coming from its triaxial shape. Contrary to the free motion, no free parameter is introduced in these computations, amplitudes and frequencies of the sinusoidal oscillations being completely determined once the orbital parameters of Eros are known, thus enabling us to calculate the solar torque. Thus we have a set of coefficients of nutations $\Delta \psi_{\text {forced }}^{\mathrm{f}}$ and $\Delta \varepsilon_{\text {forced }}^{\mathrm{f}}$, with periods very close to each other, and to half the period of rotation of the asteroid, that is to say $2.635 \mathrm{~h}$. As a consequence the combination of these oscillations results in a beating characterized by a single sinusoidal wave with varying amplitude, ranging between $0^{\prime \prime}$ and 0 ' $^{\prime} 04$ peak to peak, and with a period of $2.635 \mathrm{~h}$.

We then juxtaposed the contributions above to obtain the high frequency variations of Eros' pole of figure. They are characterized by a quasi triangular loop whose aspect slightly varies after each cycle.

Finally, we gathered all the contributions to Eros' rotation obtained analytically both in this paper and by 
Souchay et al. (2003a) to compute the two dimensional variations $(\alpha, \delta)$ of Eros' equatorial coordinates of the pole of figure. Whereas the long periodic part of these variations due to forced precession and nutation perfectly match those obtained by Miller et al. (2002) from numerical integration, it is not the case of the short periodic variations. We showed that a possible cause might be the omission in this last paper of the free rotation in obliquity.

Lastly, we have shown that Eros' polar motion is characterized by an ellipse with very large eccentricity $(e=0.8952)$ and a very fast period ( $T=0.6178 \mathrm{~d}$ ) We have also explained analytically why the variations of Eros' angular speed of rotation are negligible.

Notice that the amplitudes of the free nutattion and of the force nutations due to the triaxiality investigated here, although modelled with very good precision, are of the order of a few $\mathrm{cm}$ on the surface of Eros.

We plan to complete the present study of Eros' rotation by extending it to a very long time scale in order to determine the secular variations of the obliquity and the general precession in longitude, which should be caused by the perturbing influence of the planets. All the computations made in the present paper will be useful for that topic.
Acknowledgements. We deeply thank Mrs. Geraldine Cooke for various advices concerning the present work and for carefully checking the sentences.

\section{References}

Black, G. J., Nicholson, P. D., Bottke, \& W. F., Burns, J. A. 1999, Icarus, 140, 239

Folgueira, M., \& Souchay, J. 2005, A\&A, 432, 1102

Kinoshita, H. 1972, PASJ, 24, 423

Kinoshita, H., Hori, G., \& Nakai, H. 1974, Ann. Tokyo Astr. Obs., 14, 14

Kinoshita, H. 1977, Cel. Mech., 15, 277

Kinoshita, H. 1991, Cel. Mech., 53, 365

Konopliv, A. S., Miller, J. K., Owen, W. M., et al. 2002, Icarus, 160, 289

Miller, J. K, Konopliv, A. S., Antreasian, P. G., et al. 2002, Icarus, 155, 3

Souchay, J., Kinoshita, H., Nakai, H., \& Roux, S. 2003a, Icarus, 166, 285

Souchay, J., Folgueira, M., \& Bouquillon, S. 2003b, Earth Moon and Planets, 93, 107

Tisserand, F. 1892, Mécanique Céleste (Paris: Gauthier Villars) 